\title{
How we read: the combined use of MRI and novel PET tracers for the characterisation and treatment planning of masses in neuro-oncology
}

\author{
Arian Lasocki ${ }^{1,2^{*}}$ (i) and Rodney J. Hicks ${ }^{1,2}$
}

\begin{abstract}
Technical advances in imaging are well demonstrated by MRI (Magnetic Resonance Imaging) and PET (Positron Emission Tomography). Excellent anatomical detail and a lack of ionising radiation make MRI the standard of care for most neuroimaging indications, and advanced sequences are providing an ever-growing ability for lesion characterisation. PET utilising the tracer fluorine-18 fluorodeoxyglucose is widely used in oncology, while newer PET tracers are able to target a growing number of metabolic pathways and cell membrane receptors. The sequential use of these modalities harnesses the strengths of both, providing complementary diagnostic and therapeutic information.

Here we outline the ways in which we use MRI and PET in a complementary manner to improve lesion characterisation in neuro-oncology. Most commonly, an abnormality is detected on either PET or MRI, and the addition of the other modality allows a more confident diagnosis and/or demonstrates additional lesions, guiding treatment decisions and, in some cases, obviating the need for biopsy. These modalities may also be combined to guide the treatment of intracranial masses for which the diagnosis is known, such as neuro-endocrine tumour metastases or meningiomas refractory to conventional therapies.
\end{abstract}

Keywords: Magnetic resonance imaging, Positron emission tomography, Neuro-oncology, neuroradiology

\section{Background}

Technical advances in imaging are well demonstrated by MRI (Magnetic Resonance Imaging) and PET (Positron Emission Tomography). Excellent anatomical detail and a lack of ionising radiation make MRI the standard of care for most neuroimaging indications, while PET is widely used in oncology for diagnosis, tumour staging, post-treatment follow-up and surveillance. The most commonly utilised PET tracer, fluorine-18-fluorodeoxyglucose (FDG), has relatively limited utility as a primary diagnostic tool in neuro-oncology, however, due to high uptake in normal brain parenchyma. This limitation has been overcome by the development of newer PET tracers targeting a variety

\footnotetext{
* Correspondence: arian.lasocki@petermac.org

${ }^{1}$ Department of Cancer Imaging, Peter MacCallum Cancer Centre, Grattan St, Melbourne, Victoria 3000, Australia

${ }^{2}$ Sir Peter MacCallum Department of Oncology, The University of Melbourne, Parkville, Australia
}

(C) The Author(s). 2019 Open Access This article is distributed under the terms of the Creative Commons Attribution 4.0 International License (http://creativecommons.org/licenses/by/4.0/), which permits unrestricted use, distribution, and

of metabolic pathways or cell membrane receptors. The sequential use of these modalities harnesses the strengths of both, providing complementary information to optimise diagnosis and treatment planning. These complementary strengths have also led to the development of combined PET-MR systems, which provide improved image coregistration [1] and a lower dose of ionising radiation compared to PET-CT (Computed Tomography), with greater patient convenience.

Here we discuss the ways in which we use MRI and PET in a complementary manner to improve lesion characterisation in neuro-oncology, with illustrative clinical examples. As the cornerstone of neuroradiology, MRI provides adequate characterisation of most intracranial lesions, and advanced sequences are further increasing the information available. In selected cases, however, the addition of PET provides complementary molecular characterisation and in certain circumstances can raise 
diagnostic confidence to a level that can avoid need for confirmatory biopsy. Most commonly, an abnormality is detected on either PET-CT or MRI, and the addition of the other modality allows a more confident diagnosis. These modalities may also be combined to guide the treatment of an intracranial mass for which the diagnosis is known.

\section{Imaging protocol}

In many cases, the MRI and PET will not be reported by the same individual, thus interaction between the reporting neuroradiologist and nuclear medicine physician is important. The key factor is an understanding of how each modality may add value to the diagnostic process in particular the specific diagnoses which may take up a given PET tracer - and this guides the subsequent imaging protocol.

The MRI protocol for further characterising an abnormality seen on PET should specifically target the differentials based on the PET appearances. Volumetric pre- and post-contrast T1-weighted imaging are important, as one of the key advantages of MRI over PET is the improved anatomical delineation that fine-slice imaging provides. As standard, we also suggest axial T2-weighted imaging, FLAIR (Fluid Attenuated Inversion Recovery), DWI (diffusion-weighted imaging) and a susceptibility-sensitive sequence such as SWI (Susceptibility-Weighted Imaging), as these can provide a confident diagnosis of pathologies such as a glioma [2, 3] or pyogenic abscess [4, 5]. Perfusion-weighted imaging and spectroscopy may be added depending on the differential diagnosis based on PET.

Determining an appropriate PET tracer for further characterising an abnormality seen on MRI depends on the possible differentials based on the MRI appearances and the differential tracer uptake of these entities. Similarly, to improve characterisation of a known entity, the tracer choice will be tailored to the clinical question. Tracer choice may also be influenced by local factors such as the presence of an on-site cyclotron, but it may be possible to replace with an equivalent tracer (for example, in the case of amino acid tracers).

\section{How we read MRI and PET together in neuro- oncology}

Incidental findings on PET staging studies

Despite the relative limitations of FDG-PET in the brain, the presence of an unexpected intracranial abnormality on PET is most common on FDG-PET studies, performed either for primary staging or post-treatment re-staging. The identification of an abnormality then prompts dedicated neuroimaging such as CT or MRI to characterise the abnormality, and potentially look for additional lesions below the spatial and contrast resolution of FDG-PET. The appearance of intracranial pathology on FDG-PET is somewhat dependant on its location, as normal grey

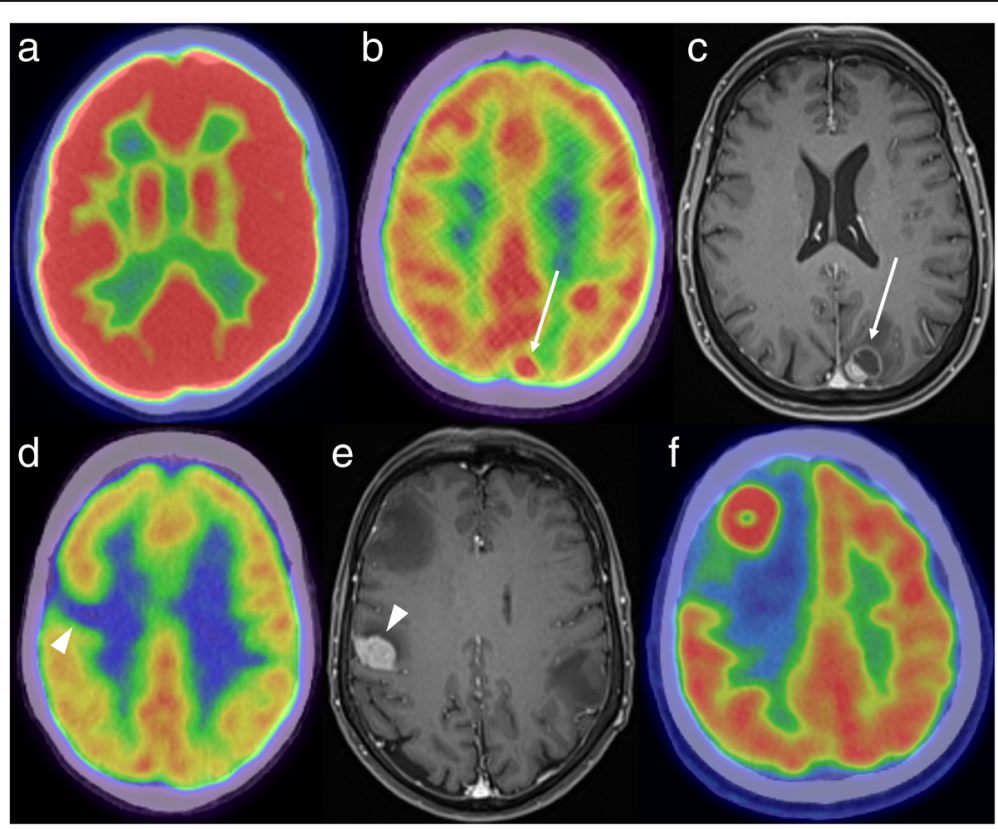

Fig. 1 FDG-PET demonstrating normal high background uptake (a) - uptake is higher in the grey matter than in the white matter. A focus of high FDG uptake in the left parietal lobe (b, white arrow) corresponds to a mixed solid/cystic metastasis on the post-contrast MRI (c). An area of low uptake (d, white arrowhead) can also be due to a metastasis, as demonstrated on the corresponding MRI (e). FDG-PET in another patient (f) shows an FDG-avid mass in the right frontal lobe with surrounding photopaenia, consistent with oedema. Histology confirmed a solitary metastasis from a lung primary 


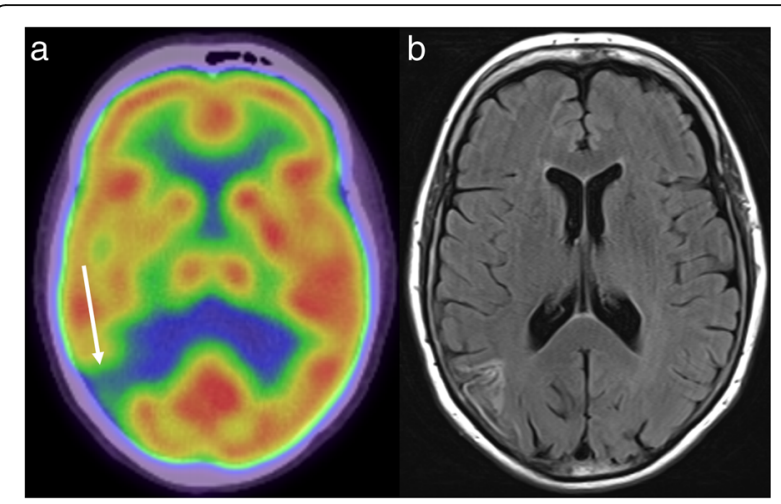

Fig. 2 FDG-PET (a) in a patient with metastatic melanoma demonstrates low uptake in the right parietal lobe (arrow). The FLAIR sequence of the corresponding MRI (b) is consistent with a previous infarct rather than a metastasis

matter has substantially higher background uptake than white matter. In some cases, intracranial pathology, such as a metastasis, is visualised as an area of high tracer uptake. Not uncommonly, however, metastases are masked by the high background uptake in the brain, especially when centred on the cortex. In these situations, a mass may instead be inferred by an area of relative photopaenia, reflecting the vasogenic oedema surrounding the mass. Sometimes, both an FDG-avid metastasis and the surrounding photopaenia may be visualised. These different appearances are illustrated in Fig. 1. MRI has particular value when PET demonstrates focal decreased uptake, as non-neoplastic aetiologies, such as an infarct, may also produce this appearance (Fig. 2).

MRI is also useful when PET staging for a primary extracranial lymphoma demonstrates secondary intracranial involvement. In this setting, MRI improves the anatomical localisation of disease and provides a better assessment of disease extent. There is particular value in identifying leptomeningeal disease, which is important clinically but often below the resolution of PET, especially when linear in morphology (Fig. 3). Other neoplasms such as high-grade gliomas are also typically FDG-avid [6], but are encountered much less frequently as an incidental finding.

Newer PET tracers targeting the somatostatin receptor are being increasingly used for the diagnosis and management of neuroendocrine tumours (NETs), such as those occurring in the pancreas or lungs, and paragangliomas (including phaeochromocytomas and extraadrenal paragangliomas). The most commonly used of these agents is gallium-68 labelled 1,4,7,10-tetraazacyclododecane-N,N',N",N"'-tetraacetic acid (DOTA)-Tyr3octreotate (also known as GaTate, DOTA-octreotate or DOTATATE). NETs are a heterogeneous group, varying by location of the primary and rate of proliferation. As such, they vary in their propensity to metastasise to the brain. For example, small cell lung carcinoma, the bestknown high-grade neuroendocrine malignancy, is frequently associated with brain metastases [7]. In contrast, paraganglioma only rarely metastasises to the brain [8].

GaTate-PET studies performed during re-staging of a NET may demonstrate an area of unsuspected tracer uptake intracranially. The differential diagnosis is based on tumours in this location which express somatostatin receptors, the main differentials being a NET metastasis, a meningioma (as meningiomas frequently express somatostatin receptors [9] and are commonly found incidentally) and a primary intracranial neoplasm that expresses somatostatin receptors, such as a haemangioblastoma [10, 11] or esthesioneuroblastoma [12]. Gliomas variably contain somatostatin receptors and are also in the differential, though the expression of somatostatin receptors is typically lower than in meningiomas $[11,13]$. In the paediatric and young adult population, the differential can be expanded to include primary embryonal tumours such as medulloblastoma $[13,14]$. In general, higher grade primary brain tumours would occur rarely as an incidental finding, other than in the setting of an underlying germline mutation. In particular, von Hippel Lindau (VHL) disease can be associated with pancreatic NET, phaeochromocytoma and

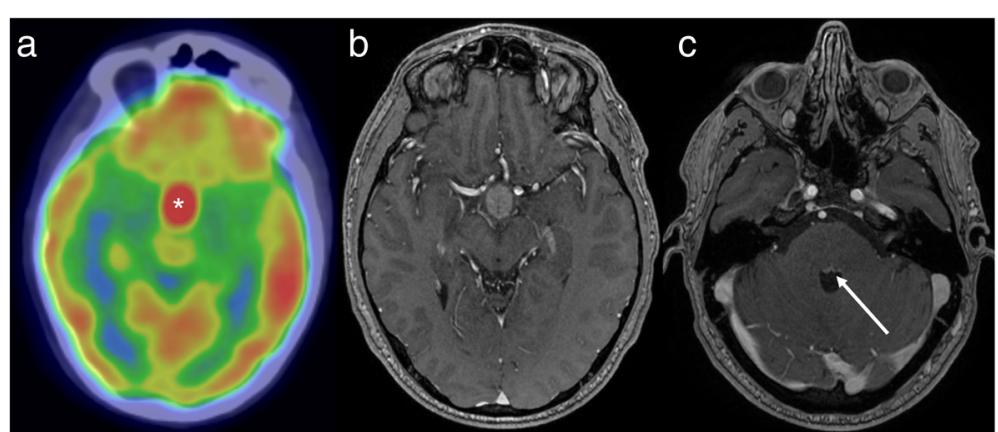

Fig. 3 FDG-PET (a) in a patient with systemic lymphoma shows abnormal intracranial uptake (asterisk), consistent with secondary CNS involvement. This is localised to the hypothalamus on the post-contrast MRI (b). The post-contrast MRI (c) also demonstrates more extensive leptomeningeal disease than is appreciable on PET, including along the ependymal surface of the fourth ventricle (arrow) 


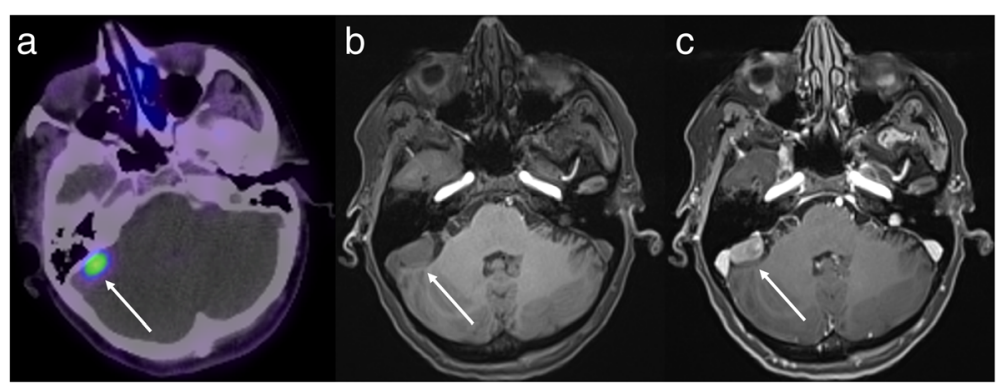

Fig. 4 GaTate-PET (a) reveals a right posterior fossa mass (arrow). The pre- and post-contrast MRI images (b and c, respectively) demonstrate a homogeneously-enhancing durally-based mass, consistent with a meningioma. This diagnosis was also supported by evidence of calcification on $\mathrm{CT}$ (not shown)

haemangioblastoma [15], all of which can express somatostatin receptors, as well as the well-recognised association with renal lesions, which do not.

MRI may then provide a specific diagnosis from this limited differential, for example by demonstrating the extra-axial location and dural tail of a meningioma (Fig. 4), cortical FLAIR hyperintensity in a glioma [2, 3], the characteristic cystic mass with a contrast-enhancing mural nodule in the case of a haemangioblastoma [10], the presence of additional lesions in a patient with metastatic disease (Fig. 5), or the olfactory groove epicentre of an esthesioneuroblastoma. GaTate-PET also plays an important role in the screening of patients with a genetic predisposition to NETs (Fig. 6), such as patients with germline SDH (succinate dehydrogenase) mutations (being predisposed to phaeochromocytomas and extraadrenal paragangliomas) [16] and, as mentioned above, von Hippel-Lindau disease [15]. Indeed, the presence of additional lesions on GaTate-PET performed for followup of patients with a solitary neuroendocrine tumour may prompt investigation for an underlying germline mutation that was previously unsuspected [10].

There has also been recent growth in the development of other targeted PET tracers. A good example is PSMA (prostate-specific membrane antigen), which has high sensitivity and specificity for the detection of prostate cancer metastases [17]. PSMA-PET may also demonstrate intracranial metastases, though this is an uncommon finding. MRI may then better demonstrate the extent of the intracranial metastatic disease, important for treatment planning. For example, surgical resection may not be feasible if MRI demonstrates more widespread metastatic disease than is visible on PET (Fig. 7).

\section{Further characterisation of a mass found on MRI}

MRI is the standard of care for the investigation of neurological symptoms and the characterisation of an abnormality identified of other imaging modalities. It can frequently suggest a specific diagnosis, but there remain cases in which the diagnosis remains uncertain. Frequently, neurosurgery is warranted for both diagnosis and treatment, but if nonoperative management is being considered depending on the diagnosis, PET may allow a more confident diagnosis without the need for craniotomy. This is particularly relevant given the growth of non-operative therapeutic techniques such as stereotactic radiosurgery or the use of systemic radionuclide therapies.

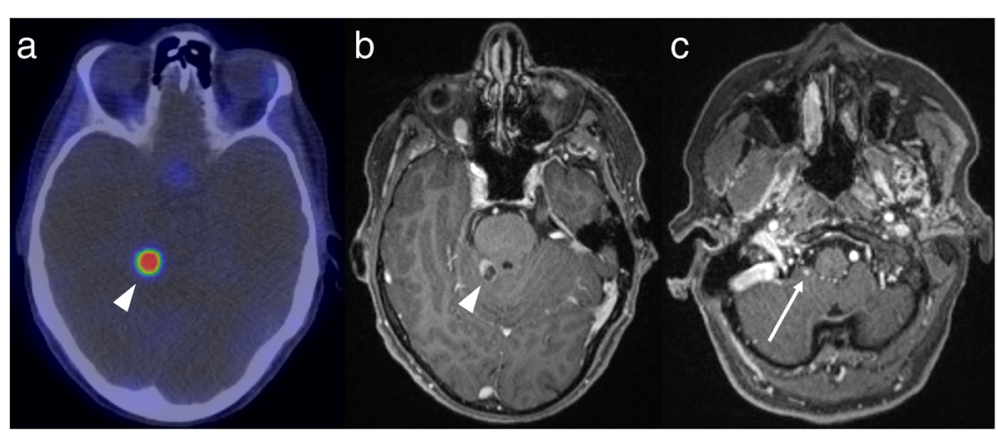

Fig. 5 GaTate-PET (a) in a patient with metastatic NET identifies a lesion in the posterior fossa (arrowhead). Given the proximity to the tentorium cerebelli, both an incidental meningioma and a NET metastasis are in the differential. The mixed solid and cystic appearance on the post-contrast MRI (b) confirms a metastasis. MRI also demonstrates a smaller enhancing focus more inferiorly in the posterior fossa (c), consistent with a further NET metastasis 


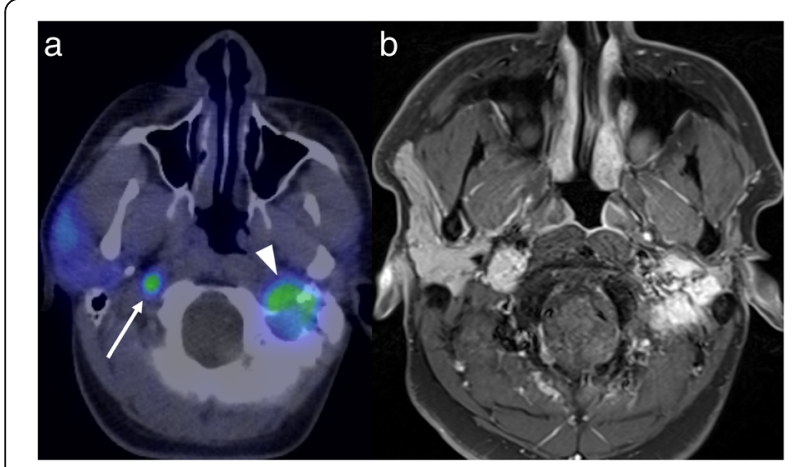

Fig. 6 FDG-PET (a) performed for follow-up of a patient with a germline succinate dehydrogenase subunit B mutation and a known left glomus jugulare paraganglioma (arrowhead) demonstrates a new area of FDG-avidity just below the skull base on the right (arrow). The subsequent post-contrast MRI (b) supports that this is a new paraganglioma rather than a metastasis

There has been a growth in the use of PET in neuroimaging due to the development of amino acid tracers such as FET (fluorine-18-fluoroethyl-L-tyrosine), MET (carbon11-methyl-L-methionine) and FDOPA (fluorine-18-fluoroL-dihydroxyphenylalanine). In contrast to FDG, these tracers do not exhibit significant uptake in normal brain parenchyma, which would otherwise limit detection and characterisation of the lesion. Amino acid PET can differentiate between intracranial neoplasms (including glioma, lymphoma and metastasis), which typically demonstrate high tracer uptake, and non-neoplastic aetiologies [6, 18]. This information, combined with conventional and advanced MRI sequences, may provide a more confident diagnosis. For example, a non-FET-avid intracranial mass has a limited differential of non-malignant conditions, including abscess [19] and tumefactive demyelination [20]. Most grade III and IV gliomas (>95\%) [21] and grade II oligodendrogliomas demonstrate high tracer uptake [18], but

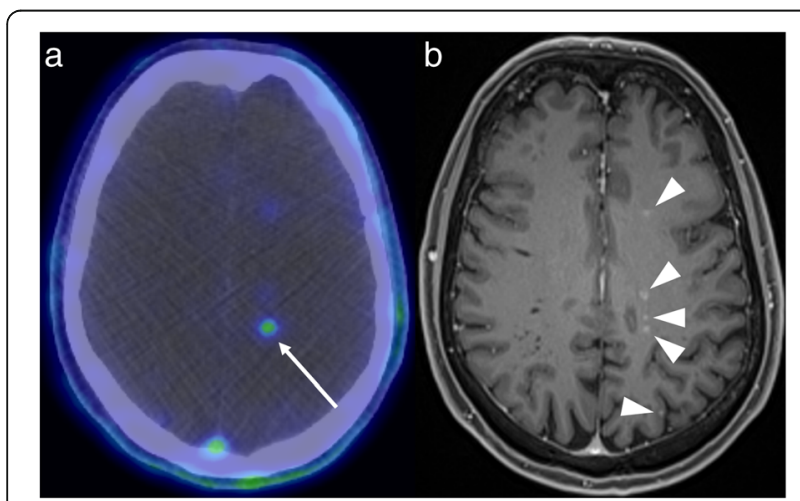

Fig. 7 PSMA-PET (a) in a patient with prostate cancer shows a focus of high uptake intracranially (arrow). The post-contrast MRI (b), however, demonstrates much more widespread intracranial metastatic disease (arrowheads) uptake is more variable in grade I and II astrocytomas, with approximately $30 \%$ exhibiting low uptake [18].

Amino acid PET has a variety of possible uses in the context of glioma, extensively outlined in a recent consensus statement [18]. At diagnosis, it can aid surgical planning, by targeting the highest uptake component for biopsy [18]. There is also a role for delineation of tumour extent prior to surgery or radiotherapy [18], which is especially relevant given recent findings that extending resection to the non-enhancing component of a glioma provides a survival benefit [22-24]. At followup, amino acid PET can help differentiate between pseudo-progression and true progression (Fig. 8), and between response and pseudo-response in patients treated with anti-antiogenic agents [18] - both scenarios being challenging for MRI even when advanced techniques are utilised. Similarly, in patients with metastatic disease treated with stereotactic radiosurgery, FET-PET is useful in distinguishing between recurrent tumour and radiation necrosis, based on differences in the tumour-tobrain uptake ratios and time-activity curves [25].

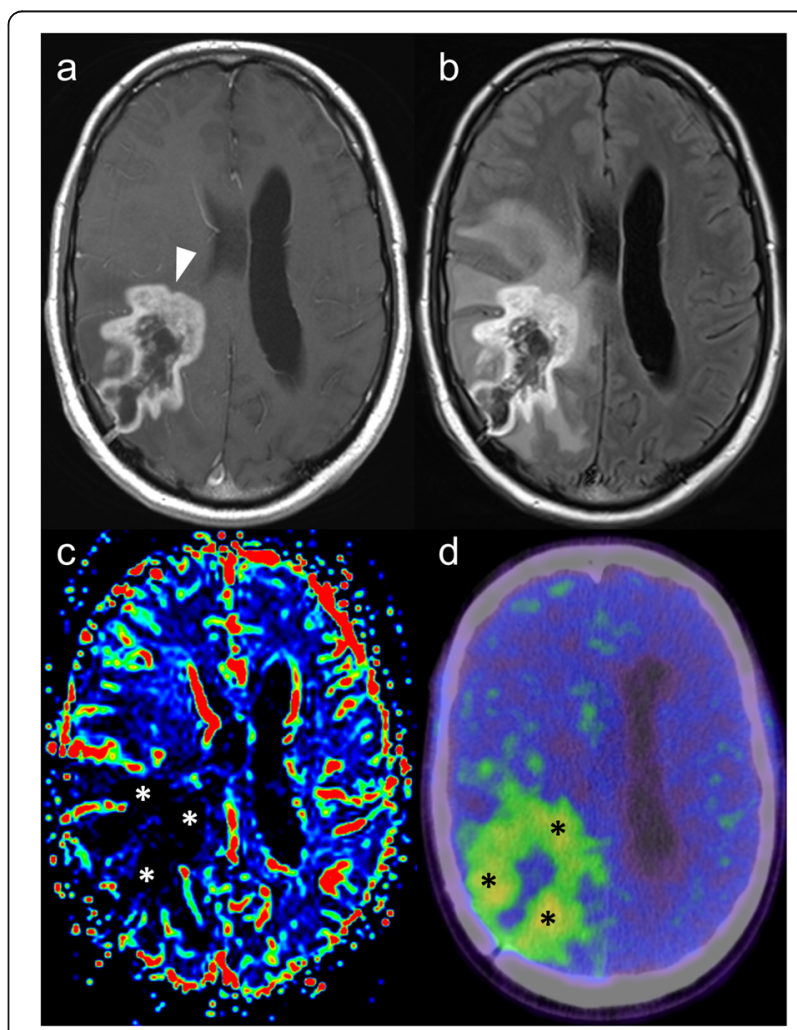

Fig. 8 Post-contrast T1-weighted (a) and FLAIR (b) MRI images demonstrate an irregular peripherally-enhancing lesion in a patient with a known right temporo-parietal glioblastoma treated with temozolamide and radiotherapy. Given an absence of elevated cerebral blood volume on dynamic susceptibility contrast MRI perfusion (c), the possibility of pseudoprogression was raised. FETPET (d) showed prominent tracer uptake, however, consistent with true tumour progression, which was confirmed histologically 


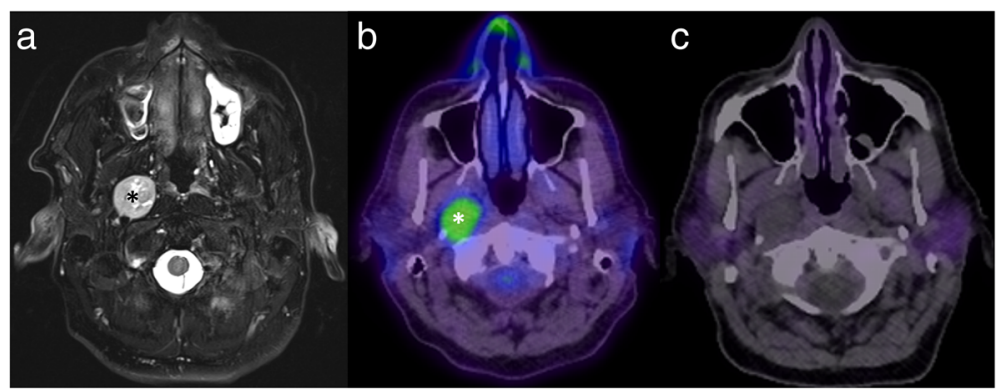

Fig. 9 Axial T2 with fat saturation MRI (a) shows a mass in the right carotid space (asterisk), slowly enlarging on serial imaging (thus going against a metastasis). There is high uptake on FDG-PET (b), but no uptake on GaTate-PET (c), most consistent with a nerve sheath tumour (confirmed histologically)

Targeted PET tracers have a potential role in the characterisation of an undifferentiated mass and the choice of tracer - and thus the potential added benefit of PET - depend on the differential diagnosis for the given lesion. For example, in the context of a mass around the skull base or within the carotid space, the main differentials to consider include a metastasis, paraganglioma and nerve sheath tumour. In this setting, the targeted nature of GaTate-PET allows the diagnosis of a paraganglioma to be either confidently diagnosed or excluded, without the risks and morbidity of open biopsy (Fig. 9). In a series examining 17 patients with metastatic phaeochromocytoma/paranglioma related to SDH type B mutations, GaTate-PET detected 285 (98.6\%) of 289 suspected metastases - higher than other functional imaging techniques and CT/MRI [26]. The lack of an optimal gold standard limits the assessment of specificity in such studies, however, as histological confirmation of small lesions not detected on other modalities is uncommon.

Another relatively common clinical situation is differentiating between a meningioma, which is commonly found incidentally, and a dural metastasis from a nonNET primary. Often, a followup MRI to demonstrate stability of a durally-based mass will be adequate. This may not be practical in the setting of known metastatic malignancy, however, in particular with primaries known to be associated with dural metastases, such as breast or prostate [27]. Similarly, the rate of growth may occasionally be greater than can be comfortably attributed to a meningioma. In such cases, GaTate-PET can be a useful problem-solver, with the presence of GaTateavidity being strong evidence of a meningioma (Fig. 10), while a metastasis is the likely diagnosis otherwise. While a previously-unidentified neuroendocrine component to the metastatic disease could provide an exception, this should be readily identifiable by the presence of GaTate uptake in other metastases. Non-GaTate-avid meningiomas are rare - in a series of 192 suspected meningiomas identified on GaTatePET and/or MRI, only two identified by MRI demonstrated no GaTate uptake, and there was no histological correlation to confirm that these were indeed false negatives on GaTate-PET [28]. The main limitation of GaTate-PET in this setting is a parasellar location, due to difficulty delineating uptake from that occurring normally in the pituitary gland [29].

Other targeted tracers, such as PSMA, can also be used in this way. For example, in a patient with a history of prostate cancer presenting with a durally-based mass, PSMA-PET could differentiate between dural metastatic disease and an incidental meningioma. Targeted PET

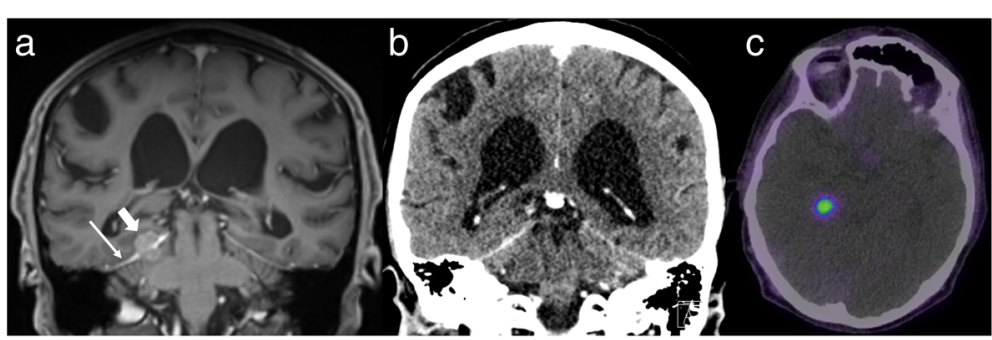

Fig. 10 Routine post-contrast MRI surveillance (a) for a patient with metastatic melanoma demonstrates a durally-based mass related to the right side of the tentorium cerebelli, best seen in the coronal plane (short arrow). The appearances are suggestive of a meningioma, but the lesion was much smaller on a CT performed only 8 months earlier (b), raising concern for a metastasis. Further characterisation with GaTate-PET (c) demonstrates high uptake, confirming the diagnosis of a meningioma rather than a metastasis 
tracers may also be useful in patients with a history of multiple malignancies presenting with intracranial metastases, allowing the histology to be determined and appropriate therapy instituted non-invasively. As new targeted PET tracers become available, this will increase the complementary value of MRI and PET.

\section{Treatment planning}

GaTate-PET also has value for treatment planning when the diagnosis is known, which is particularly relevant to the growing field of theranostics, with PET tracers being used for both diagnosis and treatment (peptide receptor radionuclide therapy, or PRRT) [30]. For example, DOTATATE can be chelated with lutetium-177 or yttrium-90 to provide radiotherapy targeted to somatostatin receptor-expressing lesions [30]. GaTate-PET is first used to predict the response to PRRT by assessing the degree of tracer uptake. Uptake is measured on the Krenning scale: $0=$ no uptake; $1=$ very low uptake; $2=$ uptake less than or equal to that of liver; $3=$ greater than liver; $4=$ greater than spleen [30, 31]. If all metastases demonstrate uptake greater than liver (Krenning 3), there is likely to be a better response to PRRT. In contrast, however, PRRT is unlikely to provide improvement

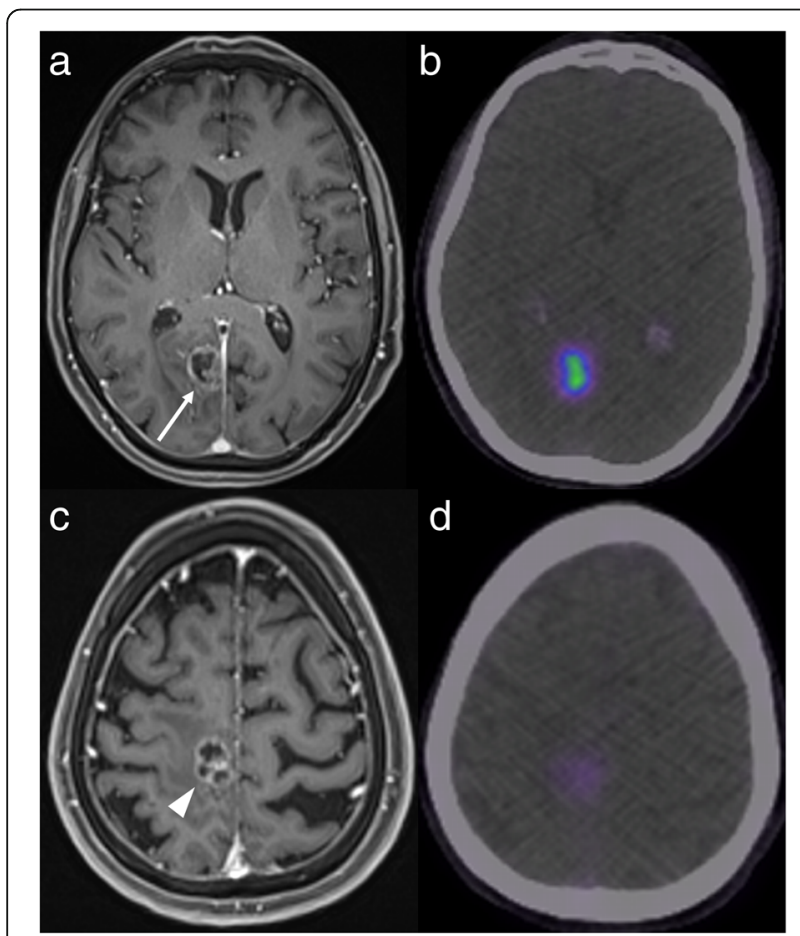

Fig. 11 Corresponding post-contrast MRI (left) and GaTate-PET (right) images of two cerebral metastases in a patient with metastatic NET. The medial right occipital metastasis (a; arrow) demonstrates high GaTate uptake (b). If this were a solitary metastasis, a response to PRRT would be expected. The medial right pre-central gyrus metastasis (c; arrowhead), however, demonstrates low GaTate uptake (d), and is unlikely to respond to PRRT

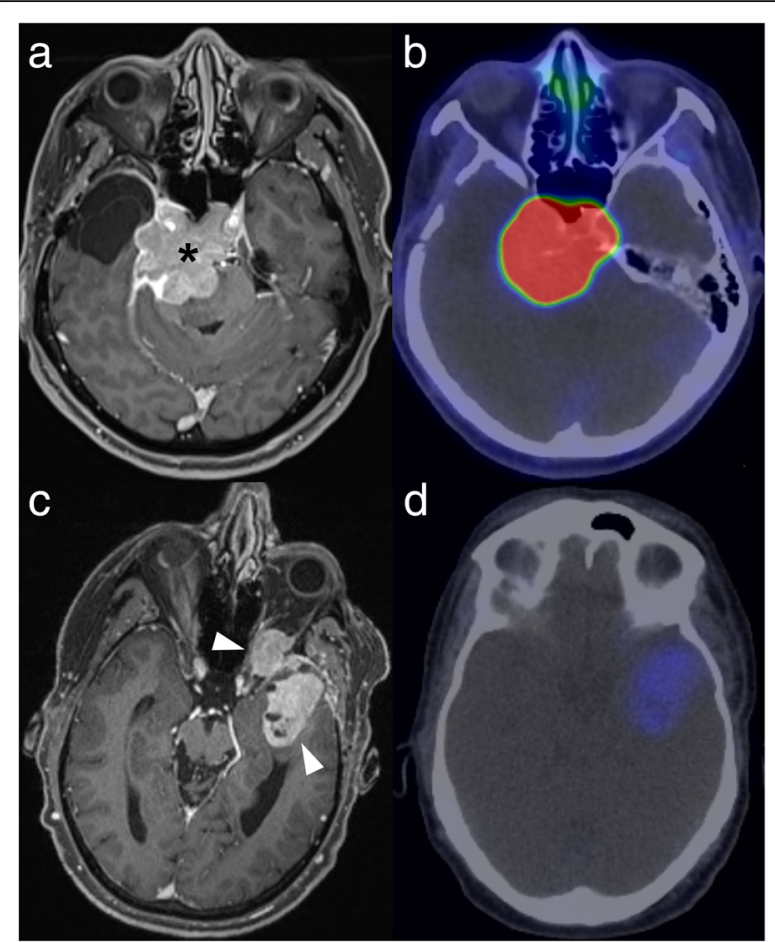

Fig. 12 Post-contrast MRI (left) and GaTate-PET (right) images of two different patients with meningiomas refractory to conventional therapy. The skull base meningioma (a; asterisk) demonstrates high GaTate uptake (b) and may benefit from PRRT. In contrast, the left temporal meningioma extending into the orbit (c; arrowheads) has only low-grade GaTate uptake (d), thus PRRT is not warranted

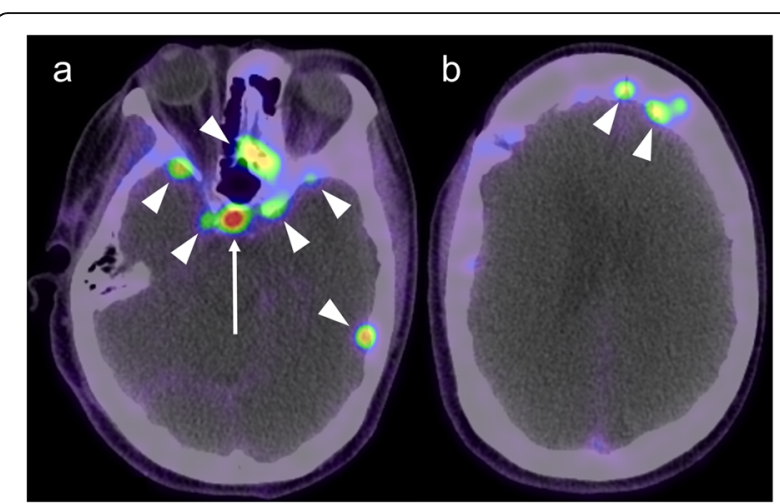

Fig. 13 Post-contrast MRI (a) and GaTate-PET (b) in a patient with previous surgery for meningioma. A small enhancing nodule related to the falx cerebri (arrows) demonstrates GaTate-avidity, consistent with meningioma. In contrast, the more diffuse dural thickening (arrowheads) does not demonstrate GaTate uptake, and is thus consistent with post-operative change rather than en plaque meningioma 


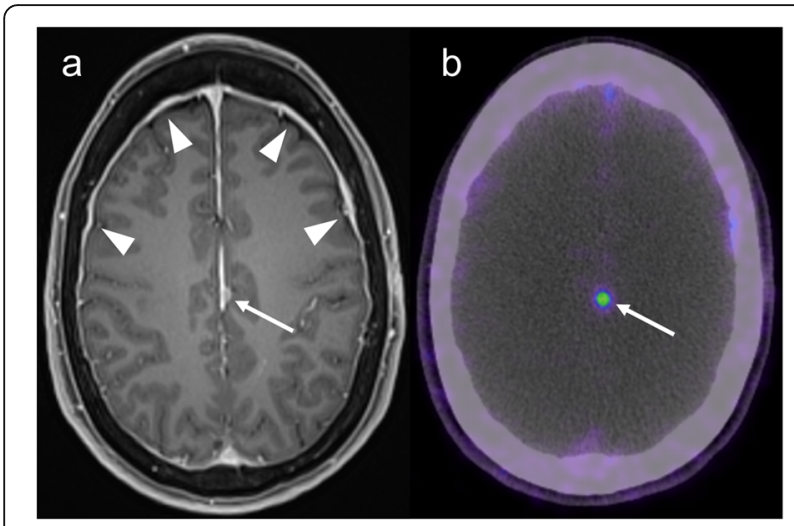

Fig. 14 GaTate-PET demonstrating multiple scattered foci of meningioma (arrowheads). Normal GaTate uptake in the pituitary gland is noted (arrow)

if uptake is Krenning 2 or less in at least one of the metastases [31] (Fig. 11). Similar principles can also be used to plan PRRT for other somatostatin receptor-expressing tumours refractory to conventional therapies, such as meningioma (Fig. 12), medulloblastoma [32] and esthesioneuroblastoma [33, 34]. There is also a role for GaTate-PET in delineating the extent of meningiomas, in particular when planning radiotherapy [28]. This is particularly useful when accurate delineation is challenging on MRI alone, for example after surgery (Fig. 13) or in the setting of en plaque or multiple meningiomas (Fig. 14).

\section{Conclusion}

MRI and PET are powerful diagnostic tools, and the complementary strengths of both can be harnessed for improving diagnostic specificity and treatment planning. This is a growing field, related to the development of novel PET tracers and the increasing utilisation of simultaneous PET-MR scanners.

\section{Abbreviations \\ CT: Computed Tomography; FDG: Fluorine-18 fluorodeoxyglucose; FDOPA: Fluorine-18-fluoro-L-dihydroxyphenylalanine; FET: Fluorine-18- fluoroethyl-L-tyrosine; GaTate: Gallium-68 labelled 1,4,7,10- tetraazacyclododecane-N,N',N",N"'-tetraacetic acid (DOTA)-Tyr3-octreotate; MET: Carbon-11-methyl-L-methionine; MRI: Magnetic Resonance Imaging; NET: Neuroendocrine tumour; PET: Positron Emission Tomography; PRRT: Peptide receptor radionuclide therapy; PSMA: Prostate-specific membrane antigen}

\section{Acknowledgments}

Nil.

\section{Authors' contributions}

Both authors contributed significantly to this manuscript and have read and approved the final manuscript.

\section{Funding}

Not applicable.
Availability of data and materials

Not applicable.

Ethics approval and consent to participate

Ethics approval was not required by our institution for this manuscript.

Consent for publication

All patient data have been anonymised.

Competing interests

AL: nil.

RH: Co-Editor-in-Chief, Cancer Imaging.

Received: 11 June 2019 Accepted: 26 July 2019

Published online: 19 August 2019

\section{References}

1. Galgano S, Viets Z, Fowler K, Gore L, Thomas JV, McNamara M, McConathy J. Practical considerations for clinical PET/MR imaging. Magn Reson Imaging Clin N Am. 2017;25:281-96.

2. Tang YM, Ngai S, Stuckey S. The solitary enhancing cerebral lesion: can FLAIR aid the differentiation between glioma and metastasis? AJNR Am J Neuroradiol. 2006;27:609-11.

3. Muccio CF, Tarantino A, Esposito G, Cerase A. Differential diagnosis by unenhanced FLAIR T2-weighted magnetic resonance images between solitary high grade gliomas and cerebral metastases appearing as contrast-enhancing cortico-subcortical lesions. J Neuro-Oncol. 2011; 103:713-7.

4. Toh CH, Wei KC, Chang CN, Hsu PW, Wong HF, Ng SH, Castillo M, Lin CP. Differentiation of pyogenic brain abscesses from necrotic glioblastomas with use of susceptibility-weighted imaging. AJNR Am J Neuroradiol. 2012;33:1534-8.

5. Antulov R, Dolic K, Fruehwald-Pallamar J, Miletic D, Thurnher MM. Differentiation of pyogenic and fungal brain abscesses with susceptibility-weighted MR sequences. Neuroradiology. 2014;56:937-45.

6. Lau EW, Drummond KJ, Ware RE, Drummond E, Hogg A, Ryan G, Grigg A, Callahan J, Hicks RJ. Comparative PET study using F-18 FET and F-18 FDG for the evaluation of patients with suspected brain tumour. J Clin Neurosci. 2010;17:43-9.

7. Castrucci WA, Knisely JP. An update on the treatment of CNS metastases in small cell lung cancer. Cancer J. 2008;14:138-46.

8. Cai P, Mahta A, Kim RY, Kesari S. Paraganglioma with intracranial metastasis: a case report and review of the literature. Neurol Sci. 2012;33:1183-4.

9. Arena S, Barbieri F, Thellung S, Pirani P, Corsaro A, Villa V, Dadati P, Dorcaratto A, Lapertosa G, Ravetti JL, et al. Expression of somatostatin receptor mRNA in human meningiomas and their implication in in vitro antiproliferative activity. J Neuro-Oncol. 2004;66:155-66.

10. Lasocki A, Akhurst T, Drummond K, Gaillard F. Cerebellar haemangioblastoma discovered incidentally on (68) Ga-DOTA-octreotate examination. Clin Neurol Neurosurg. 2016;144:20-2.

11. Dutour A, Kumar U, Panetta R, Ouafik L, Fina F, Sasi R, Patel YC. Expression of somatostatin receptor subtypes in human brain tumors. Int J Cancer. 1998;76:620-7.

12. Rostomily RC, Elias $M$, Deng $M$, Elias $P$, Born DE, Muballe $D$, Silbergeld $D L$, Futran N, Weymuller EA, Mankoff DA, Eary J. Clinical utility of somatostatin receptor scintigraphic imaging (octreoscan) in esthesioneuroblastoma: a case study and survey of somatostatin receptor subtype expression. Head Neck. 2006;28:305-12.

13. Reubi JC, Lang W, Maurer R, Koper JW, Lamberts SW. Distribution and biochemical characterization of somatostatin receptors in tumors of the human central nervous system. Cancer Res. 1987;47:5758-64.

14. Guyotat J, Champier J, Pierre GS, Jouvet A, Bret P, Brisson C, Belin MF, Signorelli F, Montange MF. Differential expression of somatostatin receptors in medulloblastoma. J Neuro-Oncol. 2001;51:93-103.

15. Maher ER, Neumann HP, Richard S. von Hippel-Lindau disease: a clinical and scientific review. Eur J Hum Genet. 2011;19:617-23.

16. Pasini B, Stratakis CA. SDH mutations in tumorigenesis and inherited endocrine tumours: lesson from the phaeochromocytoma-paraganglioma syndromes. J Intern Med. 2009;266:19-42.

17. Jilg CA, Drendel V, Rischke HC, Beck T, Vach W, Schaal K, Wetterauer U, Schultze-Seemann W, Meyer PT. Diagnostic accuracy of Ga-68-HBED-CC- 
PSMA-ligand-PET/CT before salvage lymph node dissection for recurrent prostate Cancer. Theranostics. 2017;7:1770-80.

18. Law I, Albert NL, Arbizu J, Boellaard R, Drzezga A, Galldiks N, la Fougere C, Langen KJ, Lopci E, Lowe V, et al: Joint EANM/EANO/RANO practice guidelines/SNMMI procedure standards for imaging of gliomas using PET with radiolabelled amino acids and [(18) F]FDG: version 1.0. Eur J Nucl Med Mol Imaging 2019, 46:540-557.

19. Lasocki A, Hicks RJ. Complementary role of MRI and positron emission tomography in diagnosing cerebral abscess in a patient with metastatic paraganglioma. J Med Imaging Radiat Oncol. 2018;62:525-7.

20. Kebir S, Gaertner FC, Mueller M, Nelles M, Simon M, Schafer N, Stuplich M, Schaub C, Niessen M, Mack F, et al. (18) F-fluoroethyl-L-tyrosine positron emission tomography for the differential diagnosis of tumefactive multiple sclerosis versus glioma: a case report. Oncol Lett. 2016;11:2195-8.

21. Albert NL, Weller M, Suchorska B, Galldiks N, Soffietti R, Kim MM, la Fougere C, Pope W, Law I, Arbizu J, et al. Response assessment in neuro-oncology working group and European Association for Neuro-Oncology recommendations for the clinical use of PET imaging in gliomas. NeuroOncology. 2016;18:1199-208.

22. Li YM, Suki D, Hess K, Sawaya R. The influence of maximum safe resection of glioblastoma on survival in 1229 patients: can we do better than grosstotal resection? J Neurosurg. 2016;124:977-88.

23. Pessina F, Navarria P, Cozzi L, Ascolese AM, Simonelli M, Santoro A, Clerici E, Rossi M, Scorsetti M, Bello L. Maximize surgical resection beyond contrastenhancing boundaries in newly diagnosed glioblastoma multiforme: is it useful and safe? A single institution retrospective experience. J NeuroOncol. 2017;135:129-39.

24. Lasocki A, Gaillard F. Non-contrast-enhancing tumor: a new frontier in glioblastoma research. AJNR Am J Neuroradiol. 2019;40:758-65.

25. Galldiks N, Stoffels G, Filss CP, Piroth MD, Sabel M, Ruge MI, Herzog H, Shah NJ, Fink GR, Coenen HH, Langen KJ. Role of O-(2-(18) F-fluoroethyl)-Ltyrosine PET for differentiation of local recurrent brain metastasis from radiation necrosis. J Nucl Med. 2012:53:1367-74.

26. Janssen I, Blanchet EM, Adams K, Chen CC, Millo CM, Herscovitch P, Taieb D, Kebebew E, Lehnert H, Fojo AT, Pacak K. Superiority of [68Ga]-DOTATATE PET/CT to other functional imaging modalities in the localization of SDHBassociated metastatic Pheochromocytoma and Paraganglioma. Clin Cancer Res. 2015;21:3888-95.

27. Nayak L, Abrey LE, Iwamoto FM. Intracranial dural metastases. Cancer. 2009;115:1947-53

28. Afshar-Oromieh A, Giesel FL, Linhart HG, Haberkorn U, Haufe S, Combs SE, Podlesek D, Eisenhut M, Kratochwil C. Detection of cranial meningiomas: comparison of $(6,8)$ Ga-DOTATOC PET/CT and contrast-enhanced MRI. Eur J Nucl Med Mol Imaging. 2012;39:1409-15.

29. Dittmar JO, Kratochwil C, Dittmar A, Welzel T, Habermehl D, Rieken S, Giesel FL, Haberkorn U, Debus J, Combs SE. First intraindividual comparison of contrast-enhanced MRI, FET- and DOTATOC- PET in patients with intracranial meningiomas. Radiat Oncol. 2017:12:169.

30. Hofman MS, Lau WF, Hicks RJ. Somatostatin receptor imaging with 68Ga DOTATATE PET/CT: clinical utility, normal patterns, pearls, and pitfalls in interpretation. Radiographics. 2015;35:500-16.

31. Krenning EP, Valkema R, Kooij PP, Breeman WA, Bakker WH, de Herder WW, van Eijck CH, Kwekkeboom DJ, de Jong M, Pauwels S. Scintigraphy and radionuclide therapy with [indium-111-labelleddiethyl triamine penta-acetic acid-D-Phe1]-octreotide. Ital J Gastroenterol Hepatol. 1999;31(Suppl 2):S219-23.

32. O'Dorisio MS, Khanna G, Bushnell D. Combining anatomic and molecularly targeted imaging in the diagnosis and surveillance of embryonal tumors of the nervous and endocrine systems in children. Cancer Metastasis Rev. 2008;27:665-77.

33. Sabongi JG, Goncalves MC, Alves CD, Alves J, Scapulatempo-Neto C Moriguchi SM. Lutetium 177-DOTA-TATE therapy for esthesioneuroblastoma: a case report. Exp Ther Med. 2016;12:3078-82.

34. Savelli G, Bartolomei M, Bignardi M. Somatostatin receptors imaging and therapy in a patient affected by esthesioneuroblastoma with meningeal metastases. A classic example of theranostic approach. J Neuro-Oncol. 2016; 127:617-9.

\section{Publisher's Note}

Springer Nature remains neutral with regard to jurisdictional claims in published maps and institutional affiliations.

Ready to submit your research? Choose BMC and benefit from:

- fast, convenient online submission

- thorough peer review by experienced researchers in your field

- rapid publication on acceptance

- support for research data, including large and complex data types

- gold Open Access which fosters wider collaboration and increased citations

- maximum visibility for your research: over $100 \mathrm{M}$ website views per year

At BMC, research is always in progress.

Learn more biomedcentral.com/submissions 